\title{
Crossover from classical to random-field critical exponents in As-doped $\mathrm{TbVO}_{4}$
}

\author{
C.-H. Choo, H. P. Schriemert, and D.R. Taylor \\ Department of Physics, Queen's University, Kingston, ON, Canada K7L 3N6
}

(Received November 23, 2018)

\begin{abstract}
Using birefringence techniques we have measured the critical exponents $\beta, \gamma$, and $\delta$ in As-doped $\mathrm{TbVO}_{4}$, a structural realization of the random-field Ising model where random strain fields are introduced by V-As size mismatch. For pure $\mathrm{TbVO}_{4}$ we observe the expected classical critical exponents, while for a mixed sample with $15 \%$ As concentration our results are $\beta=0.31 \pm 0.03$, $\gamma=1.22 \pm 0.07$ and $\delta=4.2 \pm 0.7$. These values are consistent with the critical exponents for the short range pure Ising model in three dimensions in agreement with a prediction by Toh. The susceptibility data showed a crossover with temperature from classical to random field critical behaviour.
\end{abstract}

Determination of the critical properties of the random field Ising modele 3 (RFIM) has shown encouraging progress recently, both experimentally and theoretically, after many years of uncertainties. Measurements on dilute antiferromagnets in a field, the most frequently studied realization of the RFIM, have been difficult to obtain because dilution of the magnetic species inhibits equilibration close to the critical temperature. However recent experiments by Slanic et al 1 on samples with only $7 \%$ dilution have shown equilibrium behaviour through the transition temperature, thus allowing confident determination of the critical exponents. In the last few years, theoretical investigations making use of a variety of analytical and computational techniques have led to predictions of the critical exponents of the RFIM that show reasonable consistency with each other. A recent analysis by Fortin and Holdsworth $\mathrm{E}$ supports earlier suggestions that the critical exponents for the RFIM in three dimensions $(d=3)$ are those of the Ising model for reduced dimension $d^{\prime}=1.5$. Nevertheless dimensional reduction has not been rigorously proved, and even if the effective dimension $d^{\prime}=1.5$ is correct the uncertainty in some of the calculated critical exponents is quite large. Likewise the experimental situation is still not satisfactory since the critical exponents measured by Slanic et al. substantial uncertainties and are only partially consistent with theory. In addition, measurements of specific heat critical exponents 6 in dilute antiferromagnets also appear to disagree with theory.

For another realization of the RFIM, where random strain fields are generated by substitutional impurities in crystals undergoing structural Ising (Jahn-Teller) transitions, the results to date have also not been conclusive. Random fields due to As/V substitutions in $\mathrm{DyVO}_{4}$, which has $d=3$ Ising exponents, appeared to increase the susceptibility exponent $\gamma$ as expected but had no effect on the order parameter exponent $\beta$. The interpretation of the effects of random fields in the As-doped $\mathrm{DyVO}_{4}$ system is complicated by the fact that the true critical behaviour of pure $\mathrm{DyVO}_{4}$ should be classical due to the long range strain coupling. However because of the relative weakness of the long range to short range interactions, classical exponents are not observable at accessible temperatures $|t| \geq 10^{-2}$, where $t=\left(T-T_{\mathrm{D}}\right) / T_{\mathrm{D}}$ is the reduced temperature, 8 leading to uncertainty on what the effects of the random fields would be. We have therefore extended these experiments to the related $\mathrm{TbVO}_{4} / \mathrm{TbAsO}_{4}$ system where the critical behayiqur of the pure compounds is unequivocally classical 1014 , and searched for changes in critical behaviour in mixed crystals due to random fields. Since this system starts from a different universality class, the results cannot be compared directly with results from dilute antiferromagnets, but it is an important system that can independently test theoretical models and predictions of random field effects. In contrast with the large number of theoretical investigations of the short-range RFIM, the literature on the random-strain version of the RFIM is very limited, consisting primarily of a paper by Toh. 15 In this paper, Toh compares the random-strain RFIM with long range forces to that of the short-range RFIM under a renormalization group analysis. The main result is that the critical exponents should change from classical values to values that are close to those of the $d=3$ pure shortrange Ising model.

The lowest $4 f$ electronic levels in $\mathrm{TbVO}_{4}$ consists of 2 singlet states $\sim 18 \mathrm{~cm}^{-1}$ apart and a non-Kramers' doublet approximately halfway between. Coupling between the doublets and lattice distortions leads to a $\mathrm{Tb}$ ion-ion interaction of the Ising form ${ }^{11}$ and a tetragonalorthorhombic phase transition at temperature $T_{D}$. Since the $\mathrm{Tb}$ ions are coupled predominantly to $k \sim 0$ acoustic phonons and to bulk strains, the ion-ion interaction is very long range. The order parameter is the macroscopic strain $a-b$ where $a$ and $b$ are basal plane unit cell parameters in the orthorhombic phase. The orthorhombic distortion gives rise to birefringence, $\Delta n$, which is proportional to $a-b$ (at least to a good approximation 10 ).

The full Hamiltonian for the coupled electron-phonon $\mathrm{TbVO}_{4}$ system in an external magnetic field can be written as

$$
H=-\frac{1}{2} \sum_{i j} J_{i j} \sigma_{i}^{z} \sigma_{j}^{z}-\frac{1}{2} \epsilon \sum_{i}\left(1+\tau_{i}^{z}\right) \sigma_{i}^{x}-\mathrm{B} \sum_{i} m_{i}^{x}
$$

where $m_{i}^{x}=\frac{1}{4} \mathrm{~g} \mu_{\mathrm{B}}\left(1+\sigma_{i}^{z}\right) \tau_{i}^{x}$ and $\sigma^{z}, \sigma^{x}, \tau^{z}$ and $\tau^{x}$ are Pauli type operators 11 16 . $J_{i j}$ describes the ion-ion interactions, $2 \epsilon$ is the high temperature splitting between the outer singlets and $\mathrm{B}$ is the magnetic field applied along 
the $x$ (or $a$ ) axis (i.e. along the 110 direction). The field $\mathrm{B}$ is able to induce an orthorhombic distortion because of the strongly anisotropic $\mathrm{Tb}$ magnetic moment in the orthorhombic phase. In the mean field approximation, a Landau expansion of the free energy, with $f=0$, shows that $\mathrm{B}^{2} / \mathrm{T}$ is effectively an ordering field, 17 and this is supported by the experimental data that follows. For B and $\epsilon$ small, $\mathrm{TbVO}_{4}$ is well described by an Ising model Hamiltonian. As the mode softening at the transition in this type of system is anisotropid 18 , classical critical exponents are expected, and observed, rather than $d=3$ Ising exponents.

In the mixed compound, $\mathrm{Tb}\left(\mathrm{As}_{x} \mathrm{~V}_{1-x}\right) \mathrm{O}_{4}$, a fraction $x$ of the $\mathrm{V}$ atoms are replaced by As atoms, generating random, static strain fields, one component of which has the right symmetry to couple to the order parameter. 19 For $\epsilon=\mathrm{B}=0$ the Hamiltonian has the form of the RFIM,

$$
H=-\frac{1}{2} \sum_{i j} J_{i j} \sigma_{i}^{z} \sigma_{j}^{z}-\sum_{i} h_{i} \sigma_{i}^{z}
$$

where $h_{i}$ is the random local strain field which is expected to have a Gaussian distribution about $h=0$. In his analysis of this type of random field system with anistropic mode softening, Toh predicts changes to the upper critical dimension, thus modifying the values of critical exponents at $d=3$.

Crystals of $\mathrm{Tb}\left(\mathrm{As}_{x} \mathrm{~V}_{1-x}\right) \mathrm{O}_{4}$ with impurity concentrations of $x=0$ and 0.15 were prepared using the flux growth method at the University of Oxford. The crystals were cut and polished perpendicular to the $c$ axis, with thickness of about $1 \mathrm{~mm}$. They were mounted in a strain-free manner with the $c$ axis horizontal in a helium optical cryostat. The crystal could be rotated about a vertical axis, allowing alignment of the $c$ axis parallel to the laser beam and one of the $a$ axes parallel to a horizontal magnetic field. A circular aperture in the sample holder of about $1 \mathrm{~mm}$ in diameter limited the sampled area to a small region of the crystal, and thus reduced effects due to any inhomogeneous composition, temperature and ordering field that may be present in the crystal.

The light source for birefringence experiments was a HeNe laser operating at $543.5 \mathrm{~nm}$, a wavelength that should give reasonable birefringence in this crystal.20 Photoelastic modulation and lock-in detection allowed sensitive measurement 21 of the phase shift $\phi$ due to the orthorhombic distortion. Adjustment of a Babinet compensator ensured that the detector output was proportional to $\phi$. In a typical experiment, the data acquisition system brought the sample to each desired temperature, waited up to 15 minutes for equilibration, and then ramped the magnetic field up and down while recording field, light intensity, and temperature.

Below $\mathrm{T}_{\mathrm{D}}$, the orthorhombic distortions are equally likely to be in the 110 or $1 \overline{1} 0$ direction, leading to the formation of twinned orthorhombic domains separated by domain walls. To avoid the cancellation of birefringence due to multiple domains, the crystal is forced into a single domain by applying a magnetic field that favours the distortion axis parallel to the magnetic field.22 23 Thus to determine the birefringence, and hence $\beta$, below $\mathrm{T}_{\mathrm{D}}$, we recorded data over a range of magnetic fields and extrapolated the high magnetic field data back to zero field. With the rather small magnetic fields available $(<0.35$ $\mathrm{T}$ ) it was sometimes difficult to achieve a single domain at temperatures close to $\mathrm{T}_{\mathrm{D}}$, at $\left|\mathrm{T}-\mathrm{T}_{\mathrm{D}}\right|<0.2 \mathrm{~K}$ for $x=0.15$, but this became progressively easier further away from $\mathrm{T}_{\mathrm{D}}$. For the $x=0$ sample where pinning is presumably weaker, single domain structure at $\mid \mathrm{T}$ $\mathrm{T}_{\mathrm{D}} \mid<0.75 \mathrm{~K}$ was relatively easy to achieve. For this reason, the critical isotherm exponent, $\delta$, obtained from the dependence of the induced birefringence on ordering field at the transition temperature, is more reliable for pure $\mathrm{TbVO}_{4}$ than that for the mixed sample. Above $\mathrm{T}_{\mathrm{D}}$, the change in induced birefringence resulting from a change in the ordering field gives the susceptibility and hence $\gamma$. Although the exponents for pure $\mathrm{TbVO}_{4}$ are known to be classical, 10 , their measurement provides a comparison with the mixed sample where modified critical exponents are expected. In experiments, $T_{D}$ appeared to vary slightly from run to run because of effects such as mounting strains and temperature gradients. To minimize the effects of a variable $T_{D}$ on the results, efforts were made to measure all exponents of one particular sample on the same run. In the power-law fits to the data, $T_{D}$ was chosen to optimize both the $\beta$ and $\gamma$ fits simultaneously. The consistency in the results of repeated experiments and the quality of the fits give confidence in the results.

The birefringence with increasing and decreasing ordering fields for various temperatures below $T_{D}$ in the $x=0.15$ sample is shown in Fig. 11. Hysteresis observed in the low field region is attributed to pinning of the multidomain structure. The data from the higher field region where hysteresis is absent were extrapolated to determine the zero field birefringence. A log-log plot of both the $x=0.15$ and $x=0$ data, fitted to a power law of the form $\Delta n \propto|t|^{\beta}$ is shown in Fig. 2. Using $\mathrm{T}_{\mathrm{D}}=29.26 \pm 0.03 \mathrm{~K}$ and data in the range $0.005<|t|<0.027$, we obtained a value of $\beta=0.31 \pm 0.03$ for the mixed sample. At larger $|t|$ we found no convincing evidence for crossover behaviour towards the classical exponent. For the pure sample, we obtained $\beta=0.46 \pm 0.06$ for $\mathrm{T}_{\mathrm{D}}=32.32 \pm 0.04$ $\mathrm{K}$ from data in the range $0.004<|t|<0.037$.

Figure 3 presents susceptibility data for selected temperatures above $T_{D}$. At temperatures close to $T_{D}$, these slopes deviate from linearity, attributable in part to the presence of non-zero birefringence at zero field, probably caused by internal strains 10 . The susceptibility exponent $\gamma$ was found by fitting the various values of $\chi(T)$ to the relation $\chi^{-1} \propto|t|^{\gamma}$. Figure 1 shows a log-log plot of the susceptibility $\chi$ versus the reduced temperature $|t|$ for $x=0$ and 0.15 samples. As can be seen from Fig. 4, the $\log$-log plot for $x=0.15$ shows two linear fits with the data closer to $T_{D}$ having a larger slope than that for data further away. Data in the range $0.027>|t|>0.01$ (closest to $\mathrm{T}_{\mathrm{D}}$ ) were optimized first to a linear fit giving 
$\mathrm{T}_{\mathrm{D}}=29.26 \pm 0.03 \mathrm{~K}$ and $\gamma=1.22 \pm 0.07$. A linear fit to data in the range $0.100>|t|>0.021$ (further from $\mathrm{T}_{\mathrm{D}}$ ) with $T_{D}$ unchanged yields a value of $\gamma=0.89 \pm 0.03$. We did not attempt to fit these data to a crossover function, but they suggest a crossover temperature near $|t|=0.024$ $(29.96 \mathrm{~K})$. The log-log plot of the susceptibility versus reduced temperature for pure $\mathrm{TbVO}_{4}$ in Fig. 1 does not show a crossover effect. A power law fit in the range $0.058>|t|>0.005$ gives $\mathrm{T}_{\mathrm{D}}=32.32 \pm 0.04 \mathrm{~K}$ and $\gamma=0.92 \pm 0.07$.

Nonlinearity in the dependence of the order parameter on the ordering field at temperatures below $\mathrm{T}_{\mathrm{D}}$ can be attributed to the prosressive detwinning of the sample with increasing field 24 (i. e. changing from a multidomain to a single domain structure). The critical isotherm exponents $\delta$ were extracted from the field-induced birefringence data at temperatures closest to that of the previously determined transition temperatures for $x=0.15$ and $x=0$ samples. Only the higher field $\Delta \mathrm{n}$ data were fitted to the power law $\left(\mathrm{B}^{2} / \mathrm{T}_{\mathrm{D}}\right)^{1 / \delta}$. For the $x=0.15$ sample, we obtained a value of $\delta=4.2 \pm 0.7$ at $T=$ $29.24 \pm 0.03 \mathrm{~K}$ and for the pure sample $\delta=2.6 \pm 0.4$ at $T=32.33 \pm 0.03 \mathrm{~K}$. The uncertainty in locating $\mathrm{T}_{\mathrm{D}}$ is incorporated in the uncertainty in $\delta$.

Our values of the critical exponents for pure $\mathrm{TbVO}_{4}$ agree satisfactorily with the values $\beta=0.5, \gamma=1$, and $\delta=3$ expected for a mean field system. For the randomfield sample our values $\beta=0.31 \pm 0.03, \gamma=1.22 \pm 0.07$, and $\delta=4.2 \pm 0.7$ are in good agreement with the exponents for the $d=3$ Ising model $25 \beta=0.33, \gamma=1.24$, and $\delta=4.8$.

Toh's predictions 15 for the effects of random fields on the exponents in this type of system are therefore well supported by our results. Toh noted that while the anisotropic strain interactions in $\mathrm{TbVO}_{4}$ reduce the upper critical dimension $d^{*}$ from 4 for the standard Ising model to 2 , resulting in classical exponents 18 , the introduction of random strain fields raises $d^{*}$ from 2 to 4 again, leaving the critical exponents the same as for the standard Ising model in $d=3$. In view of the focus in recent theoretical analysis on dimensional reduction it is of interest to examine Toh's analysis and our results in terms of changes in effective dimension $d^{\prime}$ instead of in $d^{*}$. Thus for the Ising model we regard the upper critical dimension to be fixed at 4 ; in pure $\mathrm{TbVO}_{4}$, the anisotropic strain interactions presumably raise the effective dimension from 3 to 5, giving classical exponents as before. If changes in critical properties due to random fields can be descrihed by dimensional reduction, the consensus prediction 3 国 is that random fields reduce the effective dimensionality by $2-\eta$, where $\eta$ is the exponent for the decay of magnetization coprelations. In the present case, since $\eta=0.032$ for $d=3,5$ the dimensionality should be reduced by 1.97 or essentially 2 , resulting in $d^{\prime}=3$. Hence our results, within their accuracy, are consistent with dimensionality reduction by random fields of $2-\eta \sim 2$ for this system.

* Current address: Van der Waals-Zeeman Instituut, Universiteit van Amsterdam, Valckenierstraat 65, 1018 XE Amsterdam, The Netherlands.

${ }^{1}$ D. P. Belanger and A. P. Young, J. Magn. Magn. Mater. 100, 272 (1991).

2 D. P. Belanger, in Spin Glasses and Random Fields, edited by A. P. Young (World Scientific, Singapore, 1998), p. 251.

${ }^{3}$ T. Nattermann, ibid., p. 277.

${ }^{4}$ Z. Slanic, D. P. Belanger and J. A. Fernandez-Baca, Phys. Rev. Lett. 82, 426 (1999).

${ }^{5}$ J. Y. Fortin and P. C. Holdsworth, J. Phys. A: Math. Gen. 31, 85 (1998).

${ }^{6}$ Z. Slanic and D. P. Belanger, J. Magn. Magn. Mater. 186, 65 (1998).

${ }^{7}$ K. A. Reza and D. R. Taylor, Phys. Rev. B 46, 11425 (1992).

${ }^{8}$ G. A. Gehring and M. C. Marques, J. Phys. C: Solid State Phys. 13, 3135 (1980).

${ }^{9}$ M. C. Marques, J. Phys. C: Solid State Phys. 13, 3149 (1980).

${ }^{10}$ R. T. Harley and R. M. Macfarlane, J. Phys. C: Solid State Phys. 8, L451 (1975).

${ }^{11}$ R. J. Elliott, R. T. Harley, W. Hayes and S. R. P. Smith, Proc. Roy. Soc. Lond. A 328, 217 (1972).

12 M. R. Wells and R. D. Worswick, Phys. Lett. A42, 269 (1972).

13 J. R. Sandercock, S. B. Palmer, R. J. Elliott, W. Hayes, S. R. P. Smith and A. P. Young, J. Phys. C: Solid State Phys. 5, 3126 (1972).

${ }^{14}$ W. Berkhahn, H. G. Kahle, L. Klein and H. C. Schopper, Phys. Stat. Sol.(b) 55, 265 (1973).

${ }^{15}$ H. S. Toh, J. Phys. A: Math. Gen. 25, 4767 (1992).

${ }^{16}$ G. A. Gehring and K. A. Gehring, Rep. Prog. Phys. 38, 1 (1975).

17 J. H. Page, S. R. P. Smith, D. R. Taylor and R. T. Harley, J. Phys. C: Solid State Phys. 12, L875 (1979).

${ }^{18}$ R. Cowley, Phys. Rev. B 13, 4877 (1976).

${ }^{19}$ G. A. Gehring, S. J. Swithenby and M. R. Wells, Solid State Comm. 18, 31 (1976).

${ }^{20}$ W. Hikel, H. Hess and H. G. Kahle, J. Phys: Condens. Matter 1, 2137 (1989).

${ }^{21}$ J. Ferré and G.A. Gehring, Rep. Prog. Phys. 47, 513 (1984). See arrangement (i), p. 520.

${ }^{22}$ K. A. Gehring, A. P. Malozemoff, W. Staude and R. N. Tyte, Solid State Commun. 9, 511 (1971).

${ }^{23}$ G. A. Gehring, A. P. Malozemoff, W. Staude and R. N. Tyte, J. Phys. Chem. Solids 33, 1487 (1972).

${ }^{24}$ K. A. Reza and D. R. Taylor (unpublished).

25 J. C. Le Guillou, J. Zinn-Justin, Phys. Rev. Lett. 39, 95 (1977). 


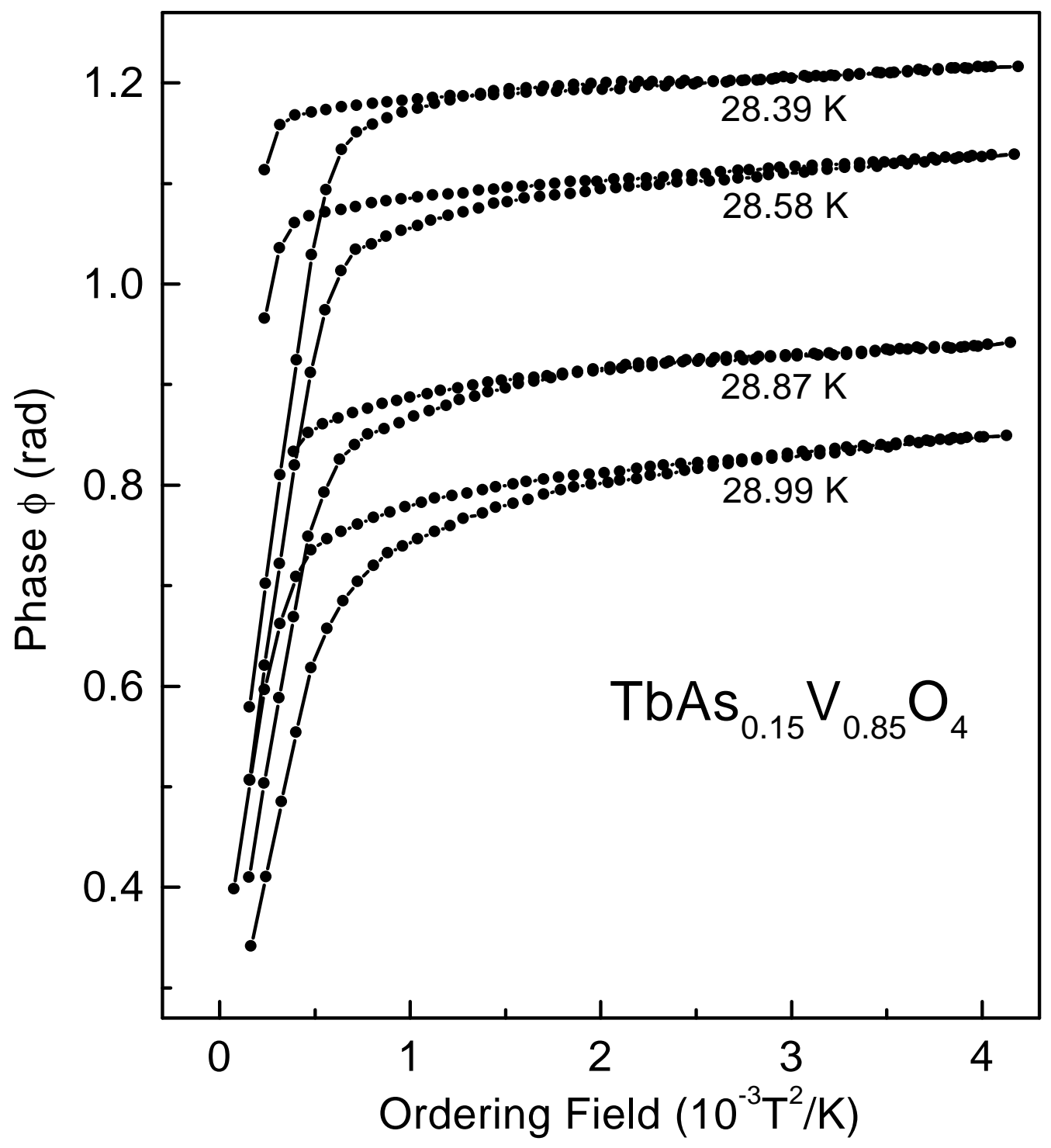

FIG. 1. Changes in phase $\phi$ (birefringence) in $x=0.15$ sample at selected temperatures below $\mathrm{T}_{\mathrm{D}}$ for increasing (lower points) and decreasing (upper points) ordering fields. The lines join data points. 


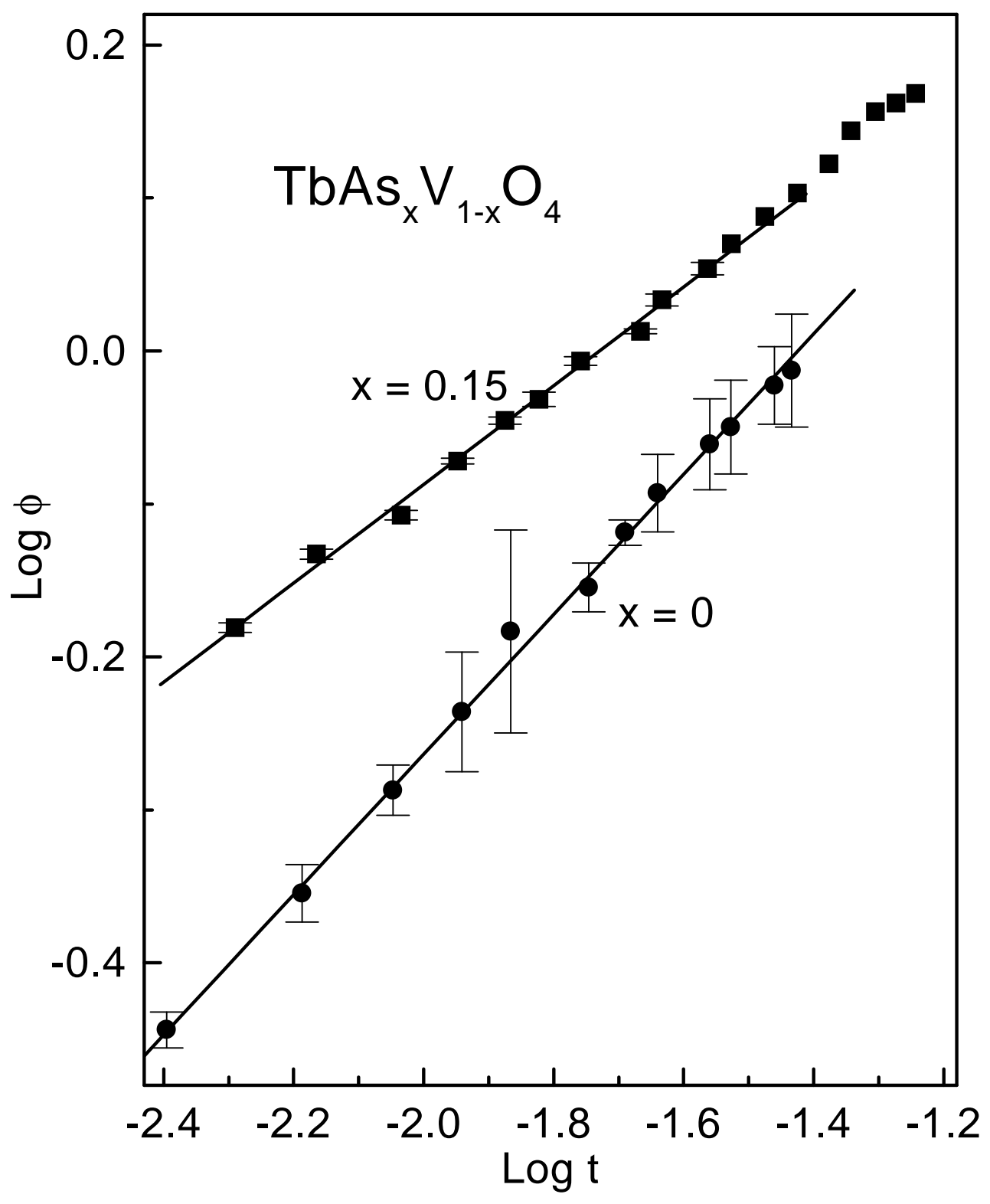

FIG. 2. Log-log plots of birefringence versus reduced temperature for $x=0$ and 0.15 . The slopes give the order parameter critical exponent $\beta$. 


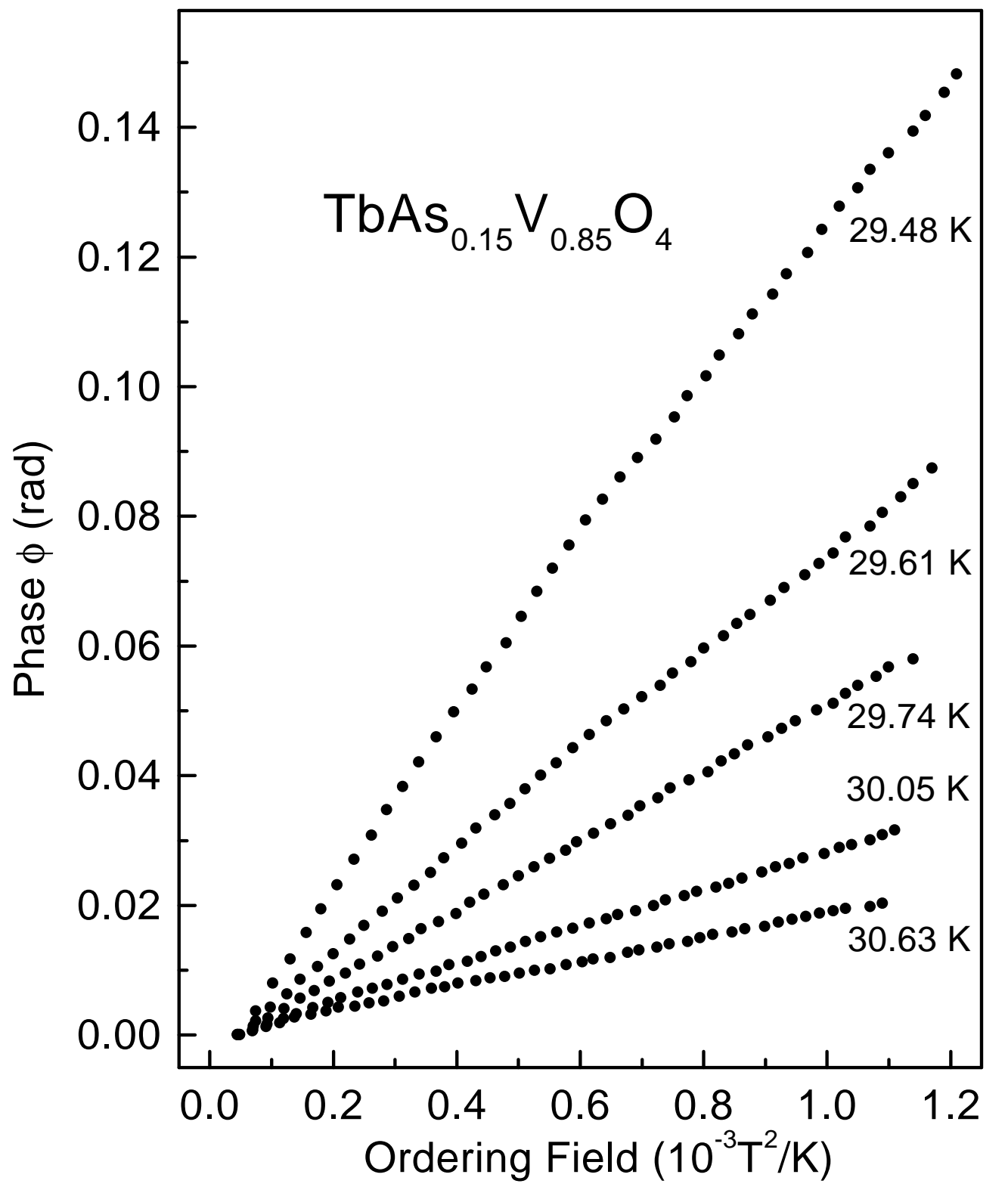

FIG. 3. Changes in birefringence versus ordering field in $x=0.15$ sample at selected temperatures above $\mathrm{T}_{\mathrm{D}}$. Slopes in the small-field limit give susceptibilities. 


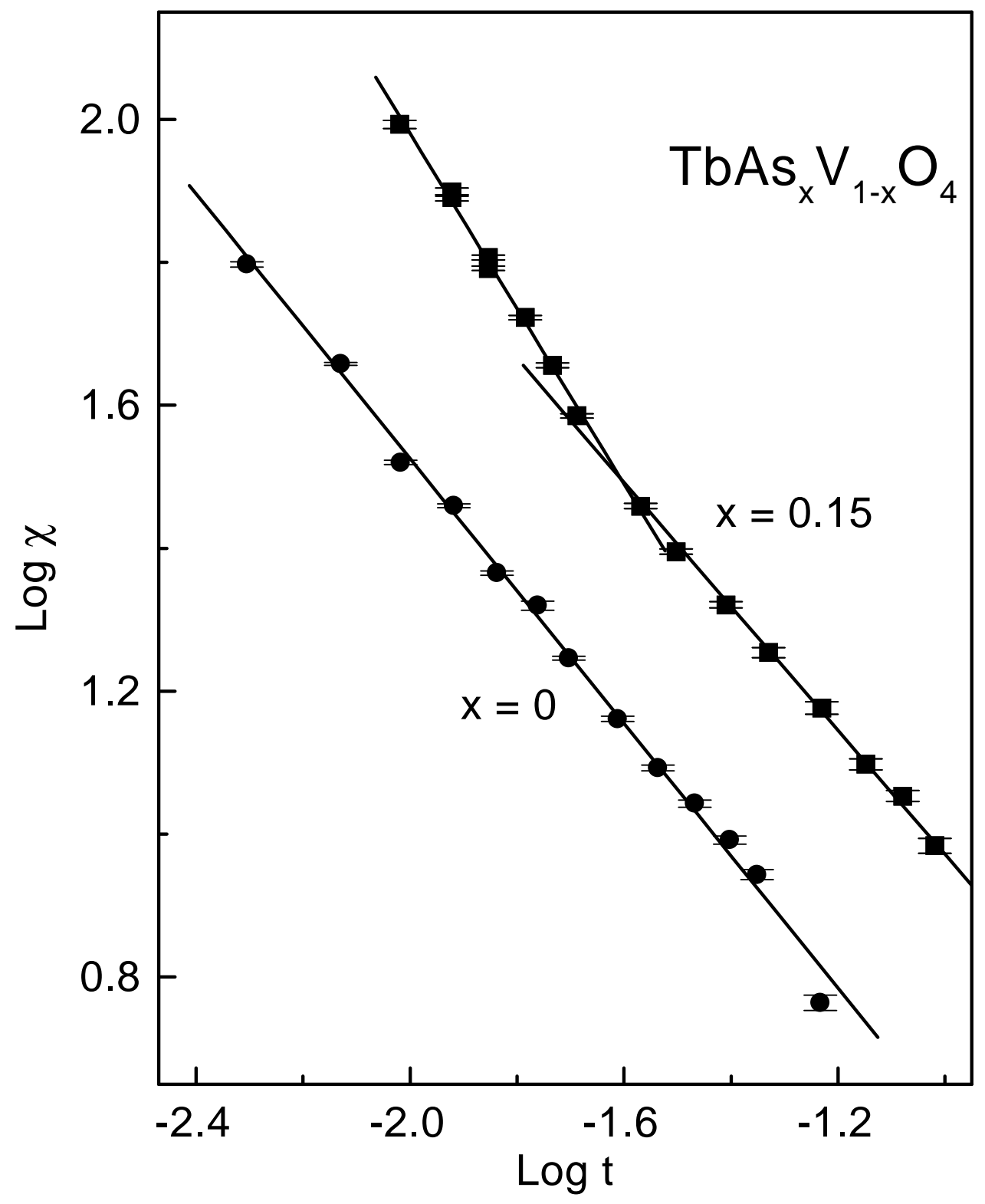

FIG. 4. Log-log plots of susceptibility versus reduced temperature for $x=0$ and 0.15 . The data for the mixed sample show a crossover from classical to random-field critical behaviour. 